\title{
Insulin resistance and pancreatic $\beta$ cell failure
}

\author{
Masato Kasuga
}

Department of Clinical Molecular Medicine, Kobe University Graduate School of Medicine, Kobe, Japan.

\begin{abstract}
It is now well accepted that diabetes mellitus is one of the main threats to human health in the twenty-first century. The total number of people with diabetes worldwide was estimated at between 151 million and 171 million in 2000 and is projected to increase to 221 million in 2010 and to 366 million in 2030 . Needless to say, the increase in the number of people with diabetes will be accompanied by an increase in the number of those with diabetic complications such as nephropathy, retinopathy, neuropathy, and atherosclerosis. The global mortality attributable to diabetes in the year 2000 was estimated at 2.9 million deaths, a number that will also increase. Given that type 2 diabetes accounts for more than $90 \%$ of cases of diabetes worldwide, it is important that we understand the pathogenesis of this condition and develop new approaches to its prevention and treatment.
\end{abstract}

\section{Development of type 2 diabetes}

In general, type 2 diabetes develops when pancreatic $\beta$ cells fail to secrete sufficient amounts of insulin to meet the metabolic demand (Figure 1). An increased metabolic demand for insulin due to insulin resistance in several tissues usually precedes the development of hyperglycemia. There is thus a period of normal or near-normal glycemia in which pancreatic $\beta$ cells compensate for insulin resistance by hypersecretion of insulin. At some point, however, this period of $\beta$ cell compensation is followed by $\beta$ cell failure, in which the pancreas fails to secrete sufficient insulin and diabetes ensues. Changes in lifestyle, such as consumption of a high-calorie diet and lack of exercise, have increased the global prevalence not only of diabetes but also of obesity. Between $60 \%$ and $90 \%$ of cases of type 2 diabetes now appear to be related to obesity (1). The insulin resistance that precedes the development of hyperglycemia is thus usually associated with obesity (Figure 1). It is therefore important to characterize the mechanisms of insulin resistance and subsequent pancreatic $\beta$ cell failure associated with obesity in order to develop approaches to prevent type 2 diabetes.

\section{Insulin resistance in specific tissues}

An understanding of insulin resistance, which is an impairment of insulin action, requires knowledge of the mechanisms of insulin action in cells. Studies with cultured cells have revealed that a signaling pathway that includes the insulin receptor, insulin receptor substrate (IRS), PI3K (class IA), phosphoinositide-dependent kinase 1 (PDK1), and the protein kinase Akt plays a central role in the metabolic actions of insulin in many cell types (2) (Figure 2). Whether or not this insulin-signaling pathway contributes to energy homeostasis in the whole body has been investigated with mouse models that harbor tissue-specific mutations in genes that encode the various pathway components.

Mice that lack insulin receptors specifically in the liver exhibit insulin resistance, glucose intolerance, and a failure of insulin to suppress hepatic glucose production and to regulate hepatic gene expression (3). A similar phenotype was demonstrated for mice in which PI3K activity was inhibited specifically in the liver as a result of the expression of a dominant negative mutant of this enzyme (4). These observations suggest that

Nonstandard abbreviations used: MCP-1, monocyte chemoattractant protein-1. Conflict of interest: The author has declared that no conflict of interest exists. Citation for this article: J. Clin. Invest. 116:1756-1760 (2006). doi:10.1172/JCI29189. insulin resistance in the liver contributes to the pathogenesis of type 2 diabetes, consistent with results obtained with cultured cells. In contrast, mice lacking insulin receptors in muscle exhibit hyperlipidemia but normal glucose tolerance (5). However, given that transgenic mice that express a dominant negative mutant of the IGF-1 receptor specifically in muscle develop insulin resistance and early-onset diabetes (6), loss of insulin signaling via insulin receptors in muscle may be compensated for by IGF-1 receptor signaling. Mice that lack insulin receptors in adipocytes are lean and are protected against obesity-related glucose intolerance (7), suggesting that insulin signaling in adipocytes per se may not contribute to the systemic insulin resistance associated with obesity.

Surprisingly, studies of mouse models with targeted mutations in genes that encode mediators of insulin signaling have suggested that such signaling in nonclassical insulin target tissues, such as the brain and pancreatic $\beta$ cells, plays an important role in the regulation of energy metabolism. The importance of insulin signaling in the central nervous system for the regulation of energy metabolism as well as for reproduction was directly demonstrated by the generation of mice that lack insulin receptors in the brain (8). Transgenic rescue of insulin receptor-deficient mice also suggested that insulin action in the brain plays a dominant role in maintenance of energy homeostasis (9). One of the actions of insulin in the brain is the suppression of hepatic glucose production (10). We previously showed that mice that lack STAT3 specifically in the liver manifest insulin resistance associated with an increase in hepatic glucose production (11). More recently, we identified signaling by IL- 6 and STAT3 in the liver as being responsible in part for the inhibition of hepatic glucose production induced by intracerebroventricular injection of insulin (12). These data thus suggest that insulin resistance in the brain may also contribute to the pathogenesis of type 2 diabetes by increasing hepatic glucose production and caloric intake. A high-fat diet has been shown to induce insulin resistance in the hypothalamus by triggering a proinflammatory response (13). In this Review series, Jens Brüning and his colleagues discuss in more detail the central actions of insulin relating to energy and glucose homeostasis (14).

The importance of insulin signaling in pancreatic $\beta$ cells was first demonstrated in IRS-2-deficient mice (15). Mice that lack insulin receptors in $\beta$ cells also have a defect in glucose sensing and a reduced $\beta$ cell mass (16). Insulin resistance in $\beta$ cells may therefore contribute to the pathogenesis of type 2 diabetes. 


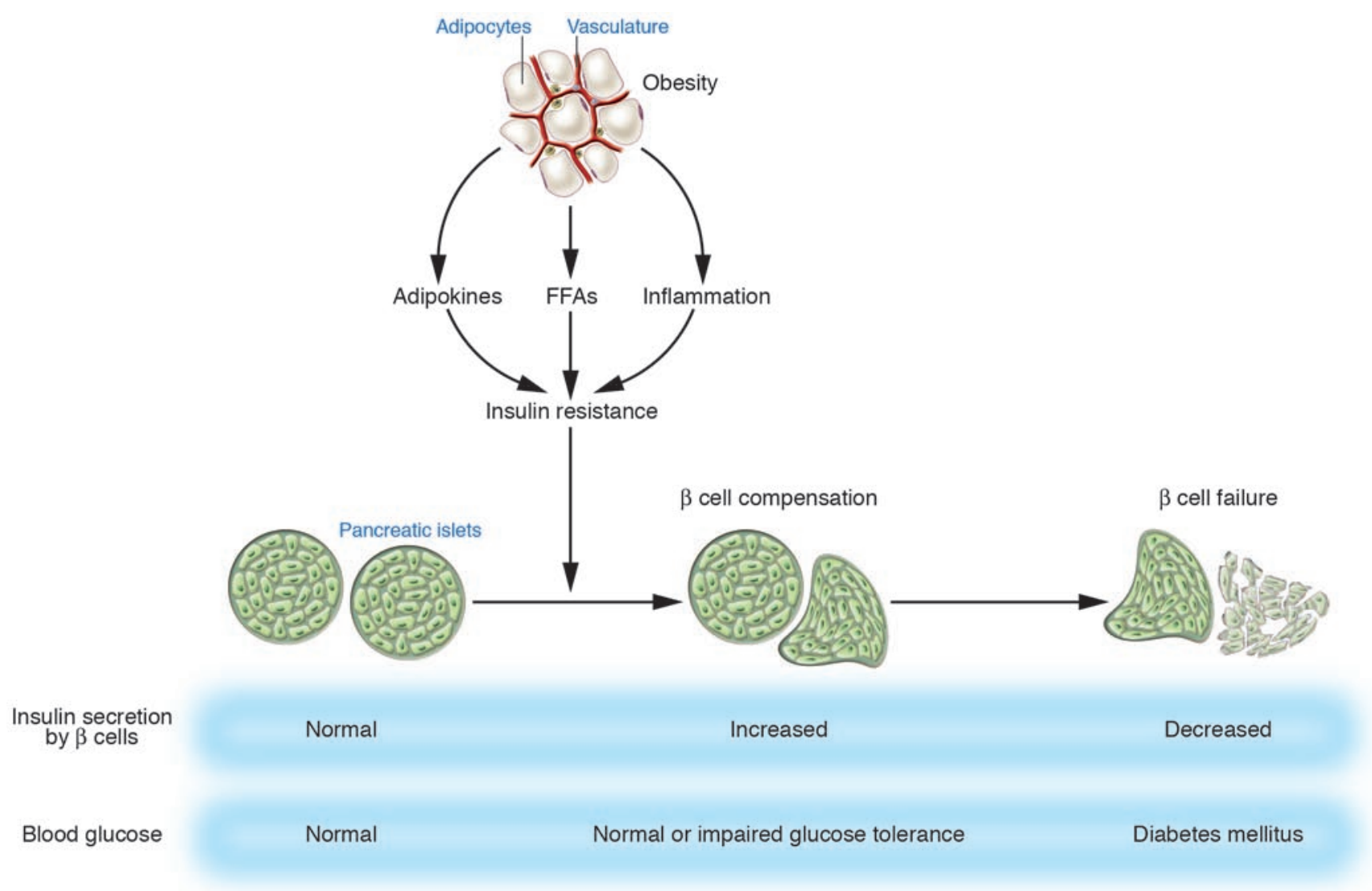

Figure 1

Development of type 2 diabetes. Insulin resistance associated with obesity is induced by adipokines, FFAs, and chronic inflammation in adipose tissue. Pancreatic $\beta$ cells compensate for insulin resistance by hypersecretion of insulin. However, at some point, $\beta$ cell compensation is followed by $\beta$ cell failure, and diabetes ensues.

\section{Systemic insulin resistance}

How does insulin resistance in the whole body develop? Studies of tissue-specific knockout mice have revealed the existence of intertissue communication (17). Such communication is mediated not only by classical hormones and the nervous system but also through the production and secretion of bioactive molecules by several cell types. It is now well established that adipocytes, for example, produce and secrete bioactive molecules known as adipokines. More recently, other types of cells including hepatocytes and myocytes have also been shown to secrete bioactive molecules such as Angptl 3 (18), Angptl 6 (19), and myostatin (20). The mechanisms by which cells sense metabolic changes and transduce such information in order to maintain energy homeostasis, with a focus on glucose sensing, are addressed in this Review series by Mark Herman and Barbara Kahn (21). In addition, Yun Chau Long and Juleen Zierath discuss the role of AMP-activated protein kinase as an energy sensor (22).

\section{Linking obesity and insulin resistance}

Adipocytes and adipose tissue are central players in the pathogenesis of insulin resistance associated with obesity. Studies into the mechanisms of insulin resistance associated with obesity have recently focused on adipokines and inflammation, which turn out to be closely related to each other.

Adipocytes are not merely a site for storage of energy in the form of triglycerides but also a source of many adipokines, some of which - including leptin, TNF- $\alpha$, resistin, adiponectin, retinol-binding protein 4 (RBP4), and monocyte chemoattractant protein-1 (MCP-1) - are implicated in the pathogenesis of insulin resistance associated with obesity in rodents. In this Review series, Takashi Kadowaki and his colleagues discuss the role of adiponectin and its receptor in insulin resistance, diabetes, and the metabolic syndrome (23). In addition, Herman and Kahn describe the role of RBP4 in insulin resistance associated with obesity (21).

Chronic inflammation of white adipose tissue characterized by macrophage infiltration $(24,25)$ is thought to contribute to insulin resistance associated with obesity. We and others have suggested that the adipokine MCP-1 and its receptor CCR2 may play a role in the recruitment of macrophages to white adipose tissue and in the initiation of an inflammatory response in mice $(26,27)$. Increased secretion of MCP-1 from adipocytes of obese mice may thus trigger such macrophage recruitment, and the infiltrated cells may in turn secrete a variety of chemokines and other cytokines that further promote a local inflammatory response and affect gene expression in adipocytes, resulting in systemic insulin resistance. In this Review series, Steven Shoelson and his colleagues discuss the link between inflammation and insulin resistance from a more general point of view (28).

The amount of adipokines secreted from adipocytes may be correlated, positively or negatively, with adipocyte size - that is, 


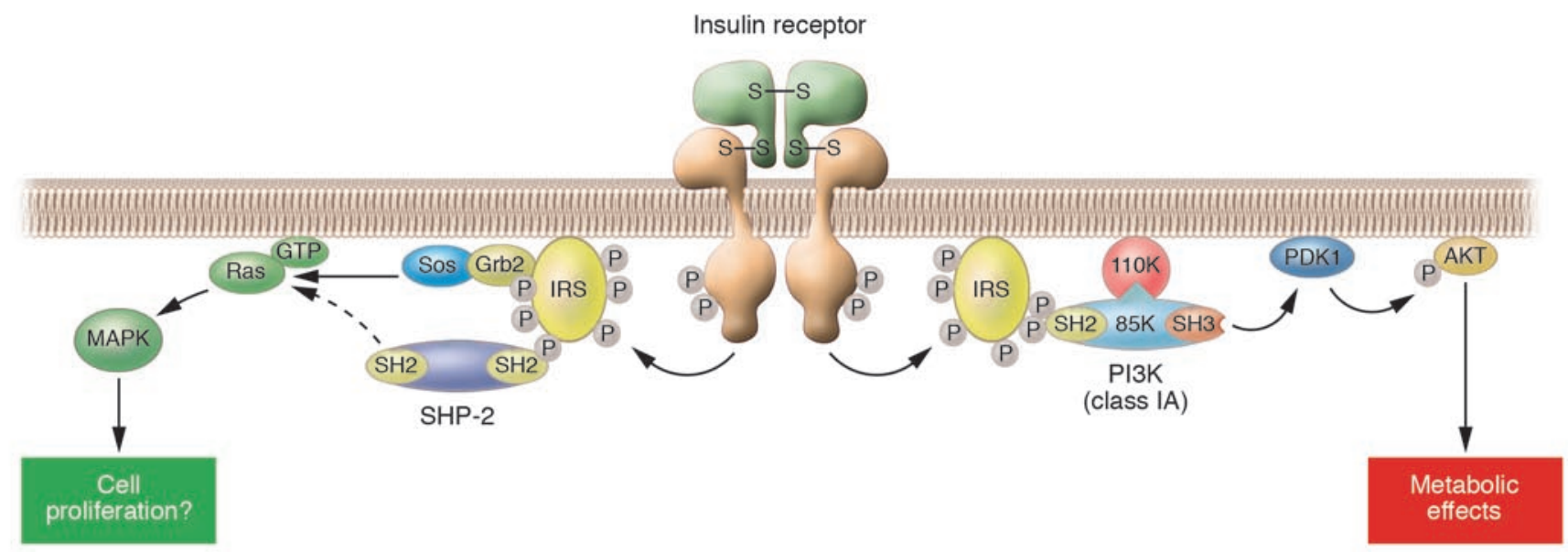

\section{Figure 2}

Insulin signaling in cells. Insulin elicits its effects by binding to its specific receptor and activating its tyrosine kinase. The activated insulin receptor kinase phosphorylates the intracellular substrate IRS, which then binds various signaling molecules containing the SH2 domain. Among them, the Grb2-Sos complex and SHP-2 transmit mitogenic signals through the activation of Ras. In contrast, PI3K (class IA) transmits the major metabolic actions of insulin via downstream effectors such as phosphoinositide-dependent kinase 1 (PDK1) and Akt.

with the amount of triglyceride stored in the cells. Such a relation implies that adipokines are direct mediators of insulin resistance associated with obesity. Given that the release of FFAs also may be correlated with adipocyte size and that an increase in the FFA concentration in plasma is a common feature of insulin resistance, FFAs are also potential mediators of insulin resistance associated with obesity. The passage of FFAs across the plasma membrane and into the cell, where they are thought to exert their effects, is mediated by a specific transport system. Fatty acid transport protein 1 (FATP1) is a transmembrane protein that enhances the cellular uptake of FFAs, and FATP1deficient mice are protected from diet-induced obesity and insulin resistance (29). The cytosol of cells also contains fatty acid-binding proteins (FABPs), which are thought to facilitate the utilization of lipids in metabolic pathways. Mice that lack both of 2 related adipocyte FABPs, aP2 and mal1, are protected from diet-induced obesity and insulin resistance (30). These observations thus suggest that the transport of FFAs into cells and their intracellular availability are important determinants of diet-induced obesity and insulin resistance. FFAs also bind to and activate members of the $G$ protein-coupled class of receptors in the plasma membrane. Among these receptors, GPR40 is preferentially expressed in pancreatic $\beta$ cells and mediates the stimulatory effect of FFAs on insulin secretion (31), and mice that lack GPR40 have a reduced susceptibility to the hyperinsulinemia, hepatic steatosis, increased hepatic glucose output, hyperglycemia, and glucose intolerance induced by obesity (32). This latter finding thus provides support for a more " $\beta$-centric" perspective of obesity-induced insulin resistance, as opposed to the "adipo-centric" perspective, with hyperinsulinemia per se possibly contributing to hepatic steatosis, hepatic insulin resistance, and hyperglycemia associated with diet-induced obesity.

It remains to be determined what kinds of signals regulate the secretion of adipokines or FFA release from adipocytes. Although oxidative stress (33) and endoplasmic reticulum-associated stress (34) are candidates for such a signal, further studies are necessary to answer this important question.

\section{Pancreatic $\beta$ cell failure}

Pancreatic $\beta$ cells initially compensate for the insulin resistance associated with obesity by upregulating the secretion of insulin. The $\beta$ cell failure and diabetes that follow this period of $\beta$ cell compensation may result from inadequate expansion of $\beta$ cell mass or failure of the existing $\beta$ cell mass to respond to glucose. Each of these 2 possible scenarios might result from a defect in insulin and IGF- 1 signaling in pancreatic $\beta$ cells. As mentioned earlier, mice that lack insulin receptors in $\beta$ cells have a defect in glucose sensing and a reduced $\beta$ cell mass (16). In contrast, mice lacking IGF- 1 receptors in $\beta$ cells manifest only a defect in glucose sensing $(35,36)$. Mice deficient in both insulin receptors and IGF-1 receptors, however, develop early-onset diabetes as a result of reduced $\beta$ cell mass (37). We also recently showed that mice with $\beta$ cells deficient in PDK1, a common downstream mediator of both insulin and IGF-1 signaling, develop a similar diabetic phenotype (38). These data suggest 2 interesting notions: (a) The phenotype associated with a disturbance in insulin and IGF- 1 signaling in $\beta$ cells may depend on the severity of the disturbance, with a virtually complete elimination of such signaling resulting in a substantial decrease in $\beta$ cell mass and a less severe impairment resulting in a decrease in the insulin secretory response to glucose. (b) Both of the prominent features of type 2 diabetes - insulin resistance in peripheral tissues and $\beta$ cell failure - may result from a defect in insulin signaling. Interestingly, another such unifying hypothesis was recently proposed: that mitochondrial dysfunction may cause both insulin resistance in peripheral tissues and impairment of glucose-induced insulin secretion in $\beta$ cells (39).

We hypothesize that the reduced pancreatic $\beta$ cell mass apparent in diabetic mice with insulin resistance is due to an impairment in cell cycle progression. Progression of the cell cycle in mammals is governed by various complexes of cyclins and cyclindependent kinases (CDKs) and by their inhibitors. We found that the CDK inhibitor $\mathrm{p} 27^{\mathrm{Kip} 1}$ progressively accumulates in the nucleus of pancreatic $\beta$ cells in IRS-2-deficient (Irs $2^{-/-}$) mice and $d b / d b$ mice (40). Deletion of the gene encoding $\mathrm{p} 27^{\mathrm{Kip} 1}$ ameliorated hyperglycemia in these animals by inducing the proliferation 
of $\beta$ cells. These observations suggest that the reduced $\beta$ cell mass and $\beta$ cell failure in Irs $2^{-/-}$or $d b / d b$ mice are due, at least in part, to

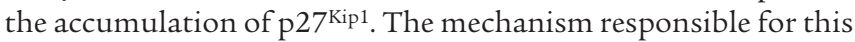
accumulation of $\mathrm{p}^{27^{\mathrm{Kip}} 1}$ in the nucleus of pancreatic $\beta$ cells of diabetic mice with insulin resistance remains to be determined. However, given that the $\mathrm{PI} 3 \mathrm{~K} / \mathrm{Akt}$ signaling pathway regulates the nuclear abundance and localization of $\mathrm{p} 27^{\mathrm{Kip} 1}$, the reduced Akt activity apparent in the islets of these mice may explain, at least in part, the accumulation of $\mathrm{p}^{27^{\mathrm{Kip}} 1}$ in the nucleus of $\beta$ cells. Furthermore, given that hyperglycemia and reactive oxygen spe-

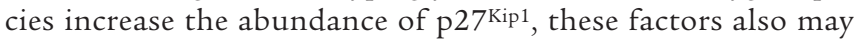
contribute to its nuclear accumulation in $\beta$ cells.

Pancreatic $\beta$ cells are exposed during $\beta$ cell compensation to metabolic changes associated with obesity, so factors commonly associated with obesity - such as insulin resistance (including that in $\beta$ cells), adipokines, FFAs, reactive oxygen species, and endoplasmic reticulum-associated stress - should therefore be examined as candidates for inducers of $\beta$ cell failure. The mechanism of $\beta$ cell failure in type 2 diabetes is extensively discussed by Mark Prentki and Christopher Nolan in this Review series (41).

\section{Type 2 diabetes and atherosclerosis}

Diabetes mellitus is a major independent risk factor for cardiovascular disease. Several studies have shown that diabetes is associated with a 2- to 4-fold increase in the risk of coronary heart disease, whereas another study concluded that diabetic patients without previous myocardial infarction are at as high a risk for myocardial infarction as are nondiabetic patients with previous myocardial infarction (42). Diabetic patients usually manifest not only hyperglycemia but also insulin resistance. In his Review, Clay Semenkovich describes data on insulin resistance in cells directly relevant to atherosclerosis and discusses the role of insulin resis- tance in the development of atherosclerosis associated with obesity-related diabetes (43). Given that systemic insulin resistance is thought to give rise to risk factors for cardiovascular disease such as hyperlipidemia and hypertension, it is difficult to dissect a role for hyperglycemia and systemic insulin resistance in the pathogenesis of atherosclerosis. The issue is further complicated by the fact that the development of atherosclerosis is itself a complex process that affects multiple target tissues. Systemic insulin resistance can be viewed as an admixture of impaired insulin action in certain target cells and excessive insulin action in others. Moreover, as type 2 diabetes progresses, the relative roles of insulin resistance versus hyperglycemia may vary, as has been highlighted by 2 recent studies of the effects of insulin receptor ablation in macrophages $(44,45)$. The apparent discrepancy between the findings of these 2 studies may be attributable to the fact that the authors measured different aspects of atherosclerotic lesions at different stages of the disease (advanced versus early) in different animal models. Epidemiological data, most recently from the Epidemiology of Diabetes Interventions and Complications (EDIC) study (46), have shown that the benefits of tight glycemic control in individuals with diabetes and atherosclerosis are somewhat less than expected, suggesting that additional risk factors (insulin resistance?) need to be considered in the prevention or treatment of atherosclerosis. This is an area where more collaboration between diabetes and atherosclerosis investigators is sorely needed in order to develop robust models of diabetic atherosclerosis in mice (47).

Address correspondence to: Masato Kasuga, Division of Diabetes and Digestive and Kidney Diseases, Department of Clinical Molecular Medicine, Kobe University Graduate School of Medicine, 7-5-2 Kusunokicho, Chuo-ku, Kobe 650-0017, Japan. Phone: 81-78-382-5860; Fax: 81-78-382-2084; E-mail: kasuga@med.kobe-u.ac.jp.
1. Anderson,J.W., Kendall, C.W., and Jenkins, D.J. 2003. Importance of weight management in type 2 diabetes: review with meta-analysis of clinical studies. J. Am. Coll. Nutr. 22:331-339.

2. Shepherd, P.R., Withers, D.J., and Siddle, K. 1998 Phosphoinositide 3-kinase: the key switch mechanism in insulin signaling. Biochem. J. 333:471-490.

3. Michael, M.D., et al. 2000. Loss of insulin signaling in hepatocytes leads to severe insulin resistance and progressive hepatic dysfunction. Mol. Cell. 6:87-94.

4. Miyake, K., et al. 2002. Hyperinsulinemia, glucose intolerance, and dyslipidemia induced by acute inhibition of phosphoinositide 3-kinase signaling in the liver. J. Clin. Invest. 110:1483-1491. doi:10.1172/JCI200215880.

5. Bruning, J.C., et al. 1998. A muscle-specific insulin receptor knockout exhibits features of the metabolic syndrome of NIDDM without altering glucose tolerance. Mol. Cell. 2:559-569.

6. Fernandez, A.M., et al. 2006. Functional inactivation of the IGF-I and insulin receptors in skeletal muscle causes type 2 diabetes. Genes Dev. 15:1926-1934.

7. Bluher, M., et al. 2002. Adipose tissue selective insulin receptor knockout protects against obesity and obesity-related glucose intolerance. Dev. Cell. 3:25-38.

8. Bruning, J.C., et al. 2000. Role of brain insulin receptor in control of body weight and reproduction. Science. 289:2122-2125.

9. Okamoto, H., et al. 2004. Transgenic rescue of insulin receptor-deficient mice. J. Clin. Invest. 114:214-223. doi:10.1172/JCI200421645.

10. Obici, S., Zhang, B.B., Karkanias, G., and Rossetti, L. 2002. Hypothalamic insulin signaling is required for inhibition of glucose production. Nat. Med. 8:1376-1382.

11. Inoue, H., et al. 2004. Role of STAT-3 in regulation of hepatic gluconeogenic genes and of carbohydrate metabolism in vivo. Nat. Med. 10:168-174.

12. Inoue, H., et al. 2006. Role of hepatic STAT3 in brain insulin action on hepatic glucose production. Cell Metab. 3:267-275.

13. Souza, C.T., et al. 2006. Consumption of a fat-rich diet activates a proinflammatory response and induces insulin resistance in the hypothalamus. Endocrinology. 146:4192-4199.

14. Plum, L., Belgardt, B.F., and Brüning, J.C. 2006. Central insulin action in energy and glucose homeostasis. J. Clin. Invest. 116:1761-1766. doi:10.1172/JCI29063.

15. Withers, D.J., et al. 1998. Disruption of IRS-2 causes type 2 diabetes in mice. Nature. 391:900-904.

16. Kulkarni, R.N., et al. 1999. Tissue-specific knockout of the insulin receptor in pancreatic beta cells creates an insulin secretory defect similar to that in type 2 diabetes. Cell. 96:329-339.

17. Abel, E.D., et al. 2001. Adipose-selective targeting of the GLUT4 gene impairs insulin action in muscle and liver. Nature. 409:729-733.

18. Koishi, R., et al. 2002. Angptl3 regulates lipid metabolism in mice. Nat. Genet. 30:151-157.

19. Oike, Y., et al. 2005. Angiopoietin-related growth factor antagonizes obesity and insulin resistance. Nat. Med. 11:400-408.

20. Zimmers, T., et al. 2002. Induction of cachexia in mice by systemically administered myostatin. Science. 296:1486-1488.

21. Herman, M.A., and Kahn, B.B. 2006. Glucose transport and sensing in the maintenance of glucose homeostasis and metabolic harmony. J. Clin. Invest. 116:1767-1775. doi:10.1172/JCI29027.

22. Long, Y.C., and Zierath, J.R. 2006. AMP-activated protein kinase signaling in metabolic regulation. J. Clin. Invest. 116:1776-1783. doi:10.1172/ JCI29044.

23. Kadowaki, T., et al. 2006 Adiponectin and adiponectin receptors in insulin resistance, diabetes, and the metabolic syndrome. J. Clin. Invest. 116:1784-1792. doi:10.1172/JCI29126.

24. Weisberg, S.P., et al. 2003. Obesity is associated with macrophage accumulation in adipose tissue. J. Clin. Invest. 112:1796-1808. doi:10.1172/ JCI200319246.

25. Xu, H., et al. 2003. Chronic inflammation in fat plays a crucial role in the development of obesity-related insulin resistance. J. Clin. Invest. 112:1821-1830. doi:10.1172/JCI200319451.

26. Kanda, H., et al. 2006. MCP-1 contributes to macrophage infiltration into adipose tissue, insulin resistance, and hepatic steatosis in obesity. J. Clin. Invest. 116:1494-1505. doi:10.1172/JCI26498.

27. Weisberg, S.P., et al. 2006. CCR2 modulates inflammatory and metabolic effects of high-fat feeding. J. Clin. Invest. 116:115-124. doi:10.1172/JCI24335.

28. Shoelson, S.E., Lee, J., and Goldfine, A.B. 2006. Inflammation and insulin resistance. J. Clin. Invest. 116:1793-1801. doi:10.1172/JCI29069.

29. Kim, J.K., et al. 2004. Inactivation of fatty acid transport protein 1 prevents fat-induced insulin resistance in skeletal muscle. J. Clin. Invest. 113:756-763. doi:10.1172/JCI200418917.

30. Maeda, K., et al. 2005. Adipocyte/macrophage fatty acid binding proteins control integrated metabolic responses in obesity and diabetes. Cell Metab. 
1:107-119.

31. Itoh, Y., et al. 2003. Free fatty acids regulate insulin secretion from pancreatic beta cells through GPR40. Nature. 422:173-176.

32. Steneberg, P., Rubins, N., Bartoov-Shifman, R., Walker, M.D., and Edlund, H. 2005. The FFA receptor GPR40 links hyperinsulinemia, hepatic steatosis, and impaired glucose homeostasis in mouse. Cell Metab. 1:245-258.

33. Furukawa, S., et al. 2004. Increased oxidative stress in obesity and its impact on metabolic syndrome. J. Clin. Invest. 114:1752-1761. doi:10.1172/ JCI200421625.

34. Ozcan, U., et al. 2004. Endoplasmic reticulum stress links obesity, insulin action, and type 2 diabetes. Science. 306:457-461.

35. Kulkarni, R.N., et al. 2002. Beta-cell-specific deletion of the Igf1 receptor leads to hyperinsulinemia and glucose intolerance but does not alter beta-cell mass. Nat. Genet. 31:111-115.

36. Xuan, S., et al. 2002. Defective insulin secretion in pancreatic $\beta$ cells lacking type 1 IGF receptor. J. Clin. Invest. 110:1011-1019. doi:10.1172/ JCI200215276.

37. Ueki, K., et al. 2006. Total insulin and IGF-I resistance in pancreatic $\beta$ cells causes overt diabetes. Nat. Genet. 38:583-588.

38. Hashimoto, N., et al. 2006. Ablation of PDK1 in pancreatic $\beta$ cells induces diabetes as a result of loss of $\beta$-cell mass. Nat. Genet. 38:589-593.

39. Lowell, B.B., and Shulman, G.I. 2005. Mitochondrial dysfunction and type 2 diabetes. Science. 307:384-387.

40. Uchida, T., et al. 2005. Deletion of Cdkn1b gene ameliorates hyperglycemia by maintaining compensatory hyperinsulinemia in diabetic mice. Nat. Med. 11:175-182.

41. Prentki, M., and Nolan, C.J. 2006. Islet $\beta$ cell failure in type 2 diabetes. J. Clin. Invest. 116:1802-1812. doi:10.1172/JCI29103.

42. Haffner, S.M., Lehto, S., Ronnemaa, T., Pyorala, K., and Laakso, M. 2003. Mortality from coronary heart disease in subjects with type 2 diabetes and in nondiabetic subjects with and without prior myocardial infarction. N. Engl. J. Med. 339:229-234.

43. Semenkovich, C.F. 2006. Insulin resistance and atherosclerosis. J. Clin. Invest. 116:1813-1822. doi:10.1172/JCI29024.

44. Baumgartl, J., et al. 2006. Myeloid lineage cellrestricted insulin resistance protects apolipoproteinE-deficient mice against atherosclerosis. Cell Metab. 3:247-256.

45. Han, S., et al. 2006. Macrophage insulin receptor deficiency increases ER stress-induced apoptosis and necrotic core formation in advanced atherosclerotic lesions. Cell Metab. 3:257-266.

46. DCCT/EDIC Study Research Group. 2005. Intensive diabetes treatment and cardiovascular disease in patients with type 1 diabetes. N. Engl. J. Med. 353:2643-2653.

47. Goldberg, I.J. 2004. Why does diabetes increase atherosclerosis? I don't know! J. Clin. Invest. 114:613-615. doi:10.1172/JCI200421645. 